\title{
Ross Chambers, On Inventing Unknownness. The Poetry of Disenchanted Reenchantment (Leopardi, Baudelaire, Rimbaud, Justice)
}

\section{Maria Emanuela Raffi}

\author{
(2) OpenEdition \\ Journals \\ Edizione digitale \\ URL: http://journals.openedition.org/studifrancesi/6990 \\ DOI: $10.4000 /$ studifrancesi.6990 \\ ISSN: 2421-5856 \\ Editore \\ Rosenberg \& Sellier
}

\section{Edizione cartacea}

Data di pubblicazione: 1 septembre 2010

Paginazione: 396

ISSN: 0039-2944

\section{Notizia bibliografica digitale}

Maria Emanuela Raffi, «Ross Chambers, On Inventing Unknownness. The Poetry of Disenchanted Reenchantment (Leopardi, Baudelaire, Rimbaud, Justice)», Studi Francesi [Online], 161 (LIV | II) | 2010, online dal 30 novembre 2015, consultato il 08 janvier 2021. URL: http://journals.openedition.org/ studifrancesi/6990 ; DOI: https://doi.org/10.4000/studifrancesi.6990

Questo documento è stato generato automaticamente il 8 janvier 2021.

\section{cc) (†)}

Studi Francesi è distribuita con Licenza Creative Commons Attribuzione - Non commerciale - Non opere derivate 4.0 Internazionale. 


\title{
Ross Chambers, On Inventing Unknownness. The Poetry of Disenchanted Reenchantment (Leopardi, Baudelaire, Rimbaud, Justice)
}

\author{
Maria Emanuela Raffi
}

\section{NOTIZIA}

ROSS CHAMBERS, On Inventing Unknownness. The Poetry of Disenchanted Reenchantment (Leopardi, Baudelaire, Rimbaud, Justice), «French Forum», vol. 33, nos. 1-2, Winter/Spring 2008, pp. 15-36.

Il saggio di Chambers parte dall'affermazione di Rimbaud contenuta nella lettera a Demeny del 1871 - «Les inventions d'inconnu demandent des formes nouvelles» - e si interroga sulla natura di tali invenzioni che Rimbaud, e dopo di lui Mallarmé, si sforzano di esprimere. L'accento è posto da Chambers sulla natura costruita e in qualche modo ingannatrice dell'espressione artistica, specie quando si tratti di espressione linguistica che cerca di dire l'«inexpressible». Due meccanismi, in particolare, sembrano rappresentare per Chambers la retorica 'moderna': il «languagethinning» ("assottigliamento del linguaggio" corrispondente grosso modo alla litote) e il «language-thickening» ("ispessimento del linguaggio", corrispondente, sempre secondo Chambers, all'enfasi). Come esempi di «language-thinning» il critico cita la studiata semplicità di Beckett o quella di Donald Justice, sulla cui opera poetica si sofferma a lungo, arrivando alla fine alla definizione di «disenchanted reenchantment», per cui il testo poetico si rivela come un consapevole "reincantamento", un'operazione in cui si crea il fascino 'feticistico' della poesia, sia pure attraverso l'uso artificioso e retorico della lingua. Chambers analizza in modo dettagliato l'esempio de L'Infinito di Leopardi; passa quindi alla Servante au grand cour di Baudelaire, in cui la tematica della nostalgia del passato, tema ricorrente in tutta una zona della poesia moderna, si 
realizza nella ricreazione di una presenza perduta. Passando alla poesia Au Cabaret-Vert di Rimbaud, Chambers nota il cambiamento dalla nostalgia diacronica ad una poetica della fuga puramente spaziale e sincronica. Anche se il punto di partenza è nostalgico (ritorno a Charleville), la lingua di Rimbaud mescola il domestico e l'estraneo, il conosciuto e l'inventato; dunque, più che 'scoprire' l'ignoto, progressivamente lo inventa, continuando la rivisitazione del passato espressa da Leopardi e da Baudelaire, ma aggiungendovi una "jouissance" costruttiva tutta sensuale. 